The file would not even be that thick, and it would more or less contain all the substantive work in this area and a photocopy of the Rey 15-Item stimulus page to boot. Times have changed, of course. The application of neuropsychology in medical-legal settings has led to the development of a serious science of malingering detection. Larrabee's Assessment of Malingered Neuropsychological Deficits sets the new gold standard in this genre, at least until the second edition of this book is published.

\section{REFERENCES}

Bianchini, K.J., Greve, K.W., \& Glynn, G. (2005). On the diagnosis of malingered pain-related disability: Lesson from cognitive malingering research. Spine Journal, 5, 404-417.

Hiscock, M. \& Hiscock, C.K. (1989). Refining the forced-choice method for the detection of malingering. Journal of Clinical and Experimental Neuropsychology, 11, 967-974.
Kuhn, T.S. (1962). The Structure of Scientific Revolutions. Chicago: University of Chicago Press.

Larrabee, G.J. (in press). Aggregation across multiple indicators improves the detection of malingering: Relationship to likelihood ratios. The Clinical Neuropsychologist.

Pankratz, L., Binder, L.M., \& Wilcox, L. (1987). Evaluation of an exaggerated somatosensory deficit with symptom validity testing. Archives of Neurology, 44, 798.

Pankratz, L., Fausti, A. \& Peed, S. (1975). A forced-choice technique to evaluate deafness in a hysterical or malingering patient. Journal of Consulting and Clinical Psychology, 43, 421-422.

Slick, D.J., Sherman, E.M.S., \& Iverson, G.L. (1999). Diagnostic criteria for malingered neurocognitive dysfunction: Proposed standards for clinical practice and research. The Clinical Neuropsychologist, 13, 545-561.

Vickery, C.E., Berry, D.T.R., Inman, T.H., Harris, M.J., \& Orey, S.A. (2001). Detection of inadequate effort on neuropsychological testing: A meta-analytic review of selected procedures. Archives of Clinical Neuropsychology, 16, 45-73.

\title{
A Frontal Lobe Refresher
}

DOI: $10.1017 /$ S135561770808048X

The Frontal Lobes: Development, Function and Pathology. Jarl Risberg and Jordan Grafman (Eds.). 2006. New York: Cambridge University Press. 240 pp., \$70.00 (PB)

Reviewed by Joseph R. Sadek, Ph.D., New Mexico Veteran's Administration Health Care System and University of New Mexico Department of Psychiatry, Albuquerque, New Mexico

The Frontal Lobes: Development, Function and Pathology is an edited volume comprised of chapters that are the result of presentations at the annual Advanced Studies Institute sponsored by the International Neuropsychological Society (INS) and by the Vivian Smith Foundation. The eight chapters represent multiple approaches to studying or understanding the frontal lobes, including: evolution and genetics, primate frontal lobe neuroanatomy, cognitive neuroscience models of frontal lobe functioning, microcircuit models based on single neuron recording, developmental models of frontal lobe functioning, case studies of frontal lobe lesion patients, functional neuroimaging of frontal lobe activity, and postmortem neuropathological findings in demented patients. This is a solid but not comprehensive list of approaches to understanding frontal lobe functioning, but the editors clearly state that this volume is not designed to be an exhaustive review. Rather, it is a volume that is better viewed as the state of the art of selected approaches to frontal lobe research as of about 2004, when most of the authors presented a variation of the chapter to the INS Summer Institute. With these qualifications in mind, I can say that the volume is a useful overview that will inform the reader of current and future directions in frontal lobe research.

The chapters are ordered sensibly, starting with evolution to anatomy and ending with a chapter on clinical syndromes, with developmental and theoretical aspects contained in the middle. However, because there was no clear attempt to unify the chapters, the reader can choose where to start in the book without concern that some prerequisite reading of another chapter was necessary to understand the current chapter. As much as this is an advantage for the selective reader, the lack of coherence is the main weakness of this volume. For example, there are two chapters based on primate research, but I could not find reference in either chapter to its partner in the book. Helen Barbas provides an excellent review of the afferent and efferent connections of primary frontal cortex, including a description of anatomically localizable modality specific regions and their systematic variation in laminar connectivity. XiaoJing Wang reviews the concept of microcircuits and their role in working memory. An example of a missed opportunity for integration here is that it would have been very interesting to hear these authors' opinions about what might comprise a complete functional system of visual working memory, including nonfrontal afferents to visual working memory regions and the importance of laminar architecture.

In between the two primate-focused chapters is Jordan Grafman's concise and coherent summary of eight theoretical approaches to frontal lobe functioning. He frames the summary using a "representation" versus "process" comparison of theories of frontal lobe functioning. Simplistically speaking, "representationalists" believe that the frontal lobe stores representations (especially cognitive represen- 
tations as opposed to modality specific representations). "Process" theorists believe that no representations are stored in the frontal lobe, but that the frontal lobe specializes in synthesis and "conflict resolution" without actually storing knowledge. This contrasting framework could also have made a convenient vehicle for integrating all of the chapters, because of its clarity. It would have been easy to ask each author to apply this dichotomy to their chapter's contents.

The chapters by David Loring and Kimford Meador on classic case studies of frontal lobe lesion patients and by Arne Brun and Lars Gustafson on the neuropathology of frontal lobe dementias stand out by their absence of theory compared to the above-described chapters and an emphasis on clinical presentation. For those new to the study of frontal lobe syndromes, these chapters will be educational. For most others, the chapters could serve as focused reviews. Some of the prose in the chapter on neuropathology was difficult to follow, and the chapter was replete with anecdotal descriptions without much citation. This chapter would have benefited from depictions of the pathology to supplement the text.

Chapters 1 and 5 review the important but frequently overlooked issue of how the frontal lobes change over time. Jarl Risberg summarizes evolutionary and genetic aspects of frontal lobe development across human development. The section headings are enticing (e.g., "Do humans have larger frontal lobes than other primates?"), and the subsuming text almost always satisfies the curiosity. In addition to the informative sections on the evolution of language and intellect, there is a section on genetic findings associated with frontal lobe evolution (FOX and COMT) that provide ample source for speculation about the genetic foundation of the development of Homo sapiens from older hominids. Then, Maureen Dennis describes how the frontal lobes change from birth to adulthood. She articulates a model early in the chapter that combines representational and processing functions of the frontal lobes, and then proceeds to discuss typical and atypical development of frontal lobe functioning. She describes phenylketonuria, traumatic brain injury, and cerebellar lesions (owing to its heavy frontal connections) as potential models of atypical development, although most of the examples of atypical development in the chapter come from studies of childhood traumatic brain injury. The chapter reviews the literature on typical and atypical development of each of the nine kinds of knowledge that the frontal lobe "binds" together using working memory and inhibitory control, as proposed by the model. By the end of the chapter, there is little dispute for the conclusion that, "With respect to both structure and function, the development of the prefrontal cortex is intricate and protracted."

Finally, Daniel Ragland provides a review of localization of function in prefrontal cortex during memory encoding and retrieval from a cognitive neuroscience (primarily functional neuroimaging) perspective. Some of the chapter reviews the literature in neurologically normal participants, but the centerpiece of the review is a discussion of Ragland's work in schizophrenia. With the hemispheric encoding-retrieval asymmetry (HERA) model as a backdrop, he discusses how the left inferior lateral frontal lobe (around BA 47) is involved in semantically based verbal encoding, while the right frontal (BA 9) is involved in recognition memory. The data on abnormalities in patients with schizophrenia are presented, including the interesting notion that behavioral abnormalities can be remediated but that functional activity in the frontal lobe is not remediated in the presence of improved behavior. The author also includes a review of memory theory beginning with William James and ending with contemporaries Tulving and Squire, thus providing a welcome diversion to the temporal lobes from the by now heavy focus on the frontal lobes.

Overall, this volume is written in nontechnical language that covers a wide array of approaches to understanding frontal lobe function. It is a fine book for the professional wishing a snapshot of current approaches to frontal lobe research and would be appropriate as a supplemental text for advanced undergraduate and graduate study. The lack of integration between chapters requires the reader to make connections, but it also is a volume with a collection of free-standing scholarly reviews. 